\title{
Spatial correlations in a self-repelling ring polymer
}

\author{
J. J. Prentis ${ }^{\text {a) }}$ \\ Physics Department, The University of Michigan, Ann Arbor, Michigan 48109 \\ (Received 29 June 1981; accepted 30 September 1981)
}

\begin{abstract}
The scattering structure function $S_{N}(q)$ of a large flexible ring polymer with $N$ monomers in a good solvent is calculated as a universal expansion in the variable $q^{2}\left\langle R_{G}^{2}\right\rangle$, where $\left\langle R_{G}^{2}\right\rangle$ denotes the mean square radius of gyration of the ring. To solve the interacting polymer problem, the methods of renormalized field theory are applied directly to Fixman's cluster expansion. The results are obtained using the approximation of expanding in $\epsilon \equiv 4$ - (dimension of space) and indicate that the self-repulsion has a greater influence on the large distance behavior of a ring polymer when compared with a linear polymer. In particular, we find that the radius of gyration ratio $\left\langle R_{G}^{2}\right\rangle_{\text {ring }} /\left\langle R_{G}^{2}\right\rangle$ linc is a universal quantity and to first order in $\epsilon$ is equal to 0.568 . For ideal polymers, the ratio is known to be 0.5 .
\end{abstract}

\section{INTRODUCTION}

The symmetry which characterizes critical phenomena is known as scaling or renormalization symmetry. It manifests itself in the long range spatial correlations which dominate the behavior of matter near a critical point. Much of the understanding of this symmetry is due to the renormalization group theory. ${ }^{1}$ The discovery $^{2}$ that a long random molecule has the renormalization symmetry of a certain class of critical phenomena enabled the renormalization group and Lagrangian field theory ${ }^{3,4}$ to be incorporated into the language of polymers.

The field theoretical methods provide a framework for understanding the properties of polymer molecules from first principles. Being a critical object, a complete description of the polymer is given by a set of scaling laws with each law consisting of a scaling function and critical exponents. The highly developed technology of renormalization allows one to extract a detailed description of the scaling behavior from a realistic microscopic model of interacting polymers. The result is a unified theory of polymers that can prove the existence of scaling laws and provide the methods necessary to calculate the scaling functions and exponents. The various properties exhibited by the polymer that are independent of the microscopic structure emerge naturally in the renormalization theory from the universality of the scaling functions and exponents. The existence of a calculable interaction parameter for long polymers in the renormalized theory allows for concrete predictions of virial coefficients ${ }^{5}$ and spatial correlations. ${ }^{6,7}$ These predictions cannot be realized in the nonrenormalization theories ${ }^{8}$ due to the unknown nature of the interaction parameter. Recently, Witten and Schäfer ${ }^{7}$ have formulated a renormalizable polymer theory without reference to a Lagrangian. In this approach, the Fixman cluster expansion ${ }^{9}$ is transformed into a Feynman graph expansion whose diagrammatic structure directly determines the renormalization. Other direct renormalization methods are being developed and applied by des Cloizeaux ${ }^{10}$ and by Oono, Ohta, and Freed. ${ }^{11}$

In this paper, we exploit the renormalization symmetry

\footnotetext{
a'Supported by U. S. National Science Foundation under DMR801-2867, Polymer Program.
}

of a ring polymer to study the spatial correlations between its monomers. It is an extension to the ring problem of the work done on the linear chain by Witten and Schäfer. ${ }^{7}$ A theoretical study of ring polymers may be useful for understanding the significance of the circularity constraint characterizing circular DNA molecules in biological systems. ${ }^{12,13}$ The recent synthesis of large ring polymers in the laboratory ${ }^{14,15}$ can also provide information concerning the properties of these molecules. The spatial correlations in a polymer are determined by the correlation function $\langle\rho(x) \rho(y)\rangle$, where $\rho(x)$ is the local density of monomers at $x$ and \langle\rangle indicates an equilibrium ensemble average. For a polymer with $N$ monomers, these correlations are measured in a scattering experiment at wave vector $q$ via the structure function

$$
S_{N}(q)=\int d^{3} x \int d^{3} y \exp [i q \cdot(x-y)]\langle\rho(x) \rho(y)\rangle .
$$

For a large flexible ring polymer with no interactions, it can be shown ${ }^{16}$ that

$$
S_{N}(q)=N^{2} u^{-1} \exp \left(-u^{2}\right) \int_{0}^{u} d x e^{x^{2}} .
$$

The scaled variable $u^{2}=\frac{1}{2} q^{2}\left\langle R_{G}^{2}\right\rangle$, where $\left\langle R_{G}^{2}\right\rangle$ is the mean square radius of gyration of an ideal ring polymer. For the ring polymer with interactions, such a complete solution does not exist. We consider the behavior of $S_{N}(q)$ for a large flexible self-repelling ring polymer.

The methods of renormalized field theory are used to calculate the structure function. The renormalizability of the polymer field theory leads to statements exhibiting the renormalization symmetry. For the structure function of a large ring polymer, this symmetry manifests itself in the form of a scaling law:

$$
S_{N}(q)=N^{2} f_{s}\left(B q N^{\nu}\right) \text {. }
$$

In this expression, $f_{s}(x)$ is a universal scaling function such that $f_{s}(0)=1, B$ depends on the microscopic details of the model, and $\nu$ is the universal exponent which governs the dependence of the radius of gyration on $N$ in the law $\left\langle R_{G}^{2}\right\rangle \sim N^{2 \nu}$. The methods of dimensional regularization and minimal subtraction renormalization ${ }^{17}$ are applied directly to Fixman' $s^{9}$ cluster expansion to calculate the scaling function $f_{\mathbf{s}}(x)$ and the exponent $\nu$. This renormalization scheme carries with it the neces- 
sity of expanding in $\epsilon \equiv 4-d$, where $d$ is the dimension of space. We calculate the scaling function to first order in $\epsilon$ as a universal expansion in the variable $q^{2}\left\langle R_{G}^{2}\right\rangle$. For small $q^{2}\left\langle R_{G}^{2}\right\rangle$, we find that the structure function for the self-repelling ring is smaller than the structure function for the ideal ring with the same $\left\langle R_{G}^{2}\right\rangle$. This is identical to the behavior discovered in the linear poly mer. ${ }^{7}$ The results also indicate that the deviation from the ideal behavior due to the self-repulsion is more pronounced in a ring polymer than in a chain.

We also calculate the ratio of the radius of gyration of a ring polymer to that of a linear polymer having the same number of monomers. We find that this ratio is a universal quantity and has a first order $\epsilon$ expansion of

$$
\frac{\left\langle R_{C}^{2}\right\rangle_{\text {Ing }}}{\left\langle R_{G}^{2}\right\rangle_{1 \text { lne }}}=\frac{1}{2}\left[1+\frac{13}{96} \epsilon+\mathcal{O}\left(\epsilon^{2}\right)\right] .
$$

In four dimensions, the self-repelling polymers behave ideally and we recover the known result ${ }^{18}$ of 0.5 for ideal polymers. For the self-repelling polymers in three dimensions, the first order $\epsilon$ expansion predicts the ratio to be 0.568 . We compare this value with the predictions of Monte Carlo computer studies giving $0.559^{19}$ and $0.568^{20,21}$ and an experimental prediction $^{22}$ of $0.526 \pm 0.05$ from neutron seattering.

In Sec. II, we describe the model used for the selfrepelling ring polymer and the cluster expansion of the monomer density correlation function. Section III shows how the renormalization symmetry, in the form of a scaling law, is a consequence of the renormalizability of the polymer theory. In Sec. IV, the cluster expansion is renormalized to calculate the scaling function. In Sec. V, the results for $S_{N}(q)$ and $\left\langle R_{G}^{2}\right\rangle_{\mathrm{rlng}} /\left\langle R_{G}^{2}\right\rangle_{1 \text { ine }}$ are discussed.

\section{CLUSTER EXPANSION}

A model for a flexible ring polymer in a good solvent is a self-repelling random polygon on a lattice. The lattice is taken to be cubic with a lattice spacing $l$ and embedded in a space of $d$ dimensions. A random $N$-gon in $d$ dimensions is defined by a sequence of points $r_{i}$, $i=0,1, \ldots, N$ on the lattice from $r_{0}$ to $r_{N}$ subject to the constraints $r_{0}=r_{N}$ and $\left|r_{i+1}-r_{i}\right|=l$. The self-repelling condition is represented by a short-ranged repulsive potential $v\left(r_{i}-r_{j}\right)$ acting between every pair of monomers.

The structure function for this model in a canonical ensemble at temperature $T$ is

$$
S_{N}(q)=\sum_{r} \exp (i q \cdot r)\langle\rho(0) \rho(r)\rangle,
$$

where

$$
\langle\rho(0) \rho(r)\rangle=Z_{N}^{1} \sum_{\left\{r_{i}\right\}_{N}} \exp \left[-\frac{1}{T} \sum_{i<j} v\left(r_{i}-r_{j}\right)\right] \rho(0) \rho(r) .
$$

The symbol $\sum_{\left\{r_{i}\right\}_{M}}$ stands for a summation over all the possible configurations of a random $N$-gon. The partition function $Z_{N}$ and the monomer density function $\rho(r)$ are given by

$$
Z_{N}=\sum_{\left\{r_{i}\right\}_{N}} \exp \left[-\frac{1}{T} \sum_{i<j} v\left(r_{i}-r_{j}\right)\right] \delta\left(r_{0}\right),
$$

$$
\rho(r)=\sum_{\alpha=1}^{N} \delta\left(r_{\alpha}-r\right)
$$

where $\delta(r)$ is the Kronecker delta function. To calculate the structure function, it is sufficient to consider the following constrained partition function:

$$
\begin{aligned}
W(r, N)= & \sum_{\left\{r_{i}\right\}_{N}} \exp \left[-\frac{1}{T} \sum_{i<j} v\left(r_{i}-r_{j}\right)\right] \\
& \times \sum_{\alpha=1}^{N} \delta\left(r_{\alpha}\right) \sum_{\beta=1}^{N} \delta\left(r_{\beta}-\gamma\right) .
\end{aligned}
$$

$W(r, N)$ is the weighted number of random $N$-gon configurations which pass through the points 0 and $r$. We calculate this function as a cluster expansion involving powers of the Mayer ${ }^{23}$ factors $f(r) \equiv \exp [-v(r) / T]-1$.

It is convenient to introduce another constrained partition function that is useful in the calculation of $W(r, N)$. It is defined as the weighted number of random walk configurations of $N$ steps on the lattice which begin at site 0 and end at site $r$. This is the fixed-end partition function for a linear chain and is given by

$$
Q(r, N)=\sum_{\left|r_{i}\right|_{N}}^{\prime} \exp \left[-\frac{1}{T} \sum_{i<j} v\left(r_{i}-r_{j}\right)\right] \delta\left(r_{0}\right) \delta\left(r_{N}-r\right) .
$$

The symbol $\sum_{\left(r_{i}\right\}_{N}}^{\prime}$ stands for the summation over all the possible configurations of a random walk with $N$ steps. The corresponding function for the ideal chain $(v=0)$ is denoted by $Q_{0}(r, N)$.

An alternative representation of the polymer statistics can be realized by using a grand canonical ensemble. This formulation is convenient because it leads to the representation of the polymer theory as a field theory. This provides a simplification in the formal development as well as the calculational aspects of the theory. In this ensemble, the average number of monomers on the ring is controlled by a chemical potential $-s T$. Now the quantity of interest is the Fourier-Laplace transform $\tilde{W}(q, s)$ of $W(r, N)$ :

$$
\tilde{W}(q, s)=\sum_{r} \exp (i q \cdot r) \sum_{N=0}^{\infty} e^{-s N} W(r, N) .
$$

The corresponding Fourier-Laplace transform of the function $Q(r, N)$ is denoted by $\tilde{Q}(q, s)$. The structure function $S_{N}(q)$ can be obtained from $\bar{W}(q, s)$ by inverting the Laplace transformation.

The virtue of the formulation using a grand canonical ensemble is that the cluster expansion assumes the simple form of a Feynman graph expansion. Each term in the cluster expansion of $\tilde{W}(q, s)$ is a convolution involving free chain "propagators" $\tilde{Q}_{0}(\phi, s)$ and Fourier transformed Mayer "coupling constant" factors $\tilde{f}(\phi)$. The algebraic expressions have an extremely simple graphical counterpart which literally provide a picture of the ring polymer symbolizing the constraints $r_{0}=r_{N}$ and $\left|r_{i+1}-r_{i}\right|=l$, the correlation points 0 and $r$, and the interaction between monomer pairs. A general graph of $n$th order in $\tilde{f}(p)$ is constructed from a circle representing the constraints defining a ring polymer configuration. Three points on this circle, which represent $0, r$ and $r_{0}=r_{N}$, are distinguished from the other points. Finally, there are $n$ dashed lines connecting different 
(a)<smiles>O=[PH]1CCCCCC1</smiles>

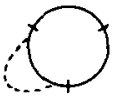

(b)

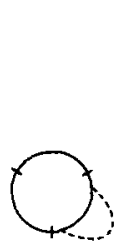

(c)

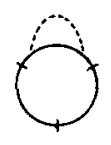

(d)

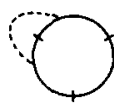

(e)

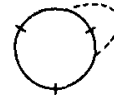

(f)

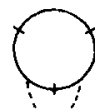

(g)
FIG. 1. The zeroth and first order diagrams for $\tilde{W}(q, s)$.

pairs of points on the circle which represent the selfrepelling interaction between monomer pairs. The $n$th order in $\tilde{f}(p)$ contribution to $\tilde{W}(q, s)$ is determined by constructing all topologically distinct graphs with $n$ interaction lines. The zeroth and first order graphs are given in Fig. 1. The evaluation of a graph proceeds according to the following rules: Label each solid line segment and each dashed line with an internal momentum variable. The external momentum $q$, conjugate to the coordinate $r$, flows out of the point $r$ and into the point 0 , representing overall momentum conservation. For each solid line carrying a momentum $k$, associate the propagator $\vec{Q}_{0}(k, s)$ and for each dashed line labeled $p$, associate the "coupling constant" factor $\tilde{f}(p)$. Finally, integrate over all the internal momenta, subject to momentum conservation at each vertex. Thus, the first order graph in Fig. 1(b) corresponds to

$$
\begin{aligned}
&\left(\frac{l}{2 \pi}\right)^{2 d} \int^{i-1} d^{d} k \int^{i-1} d \phi \tilde{f}(p) \tilde{Q}_{0}^{3}(k, s) \\
& \bar{Q}_{0}(p+k, s) \tilde{Q}_{0}(q+k, s),
\end{aligned}
$$

where $t^{-1}$ is a shorthand notation representing the domain of integration which is the Brillouin zone of a simple hypercubic lattice:

$$
\left\{p_{1}, \ldots, p_{d}|| p_{1}\left|<\frac{\pi}{l}, \ldots,\right| p_{d} \mid<\frac{\pi}{l}\right\} .
$$

If the cluster expansion is evaluated in the region which describes the large distance behavior of a ring with many monomers, then the equivalence to a Feynman graph expansion is finally established. In the grand canonical language, this critical domain is defined by $q l \ll 1$ and $0 \leqslant s-s_{c} \ll 1$, where $s_{c}$ is the critical chemical potential for which the average number of monomers becomes infinite. In this domain, the free chain function $\vec{Q}_{0}(q, s)$ has the simple form of a propagator given by

$$
\tilde{Q}_{0}(q, s)=\frac{\tau_{c}}{q^{2}+t_{0}}
$$

where

$$
\tau_{c} \equiv l^{-2} e^{s_{c}}, \quad t_{0} \equiv l^{2}\left(e^{s}-2 d\right) .
$$

A further simplification is that the Fourier transformed Mayer factor $\tilde{f}(p)$ can be set equal to $\tilde{f}(0) \equiv \tilde{f}$ since $f(r)$ is a short-ranged function. In the cluster expansion, the factors of $\tilde{f}$ appear in the combination

$$
(2 \pi)^{-d} e^{2 s} c l^{d-4} \bar{f} \equiv u \text {. }
$$

This $u$ assumes the role of the coupling constant for the unrenormalized or bare theory. Now the structure of the polymer graphs and their algebraic representation are mathematically equivalent to a Euclidean field theory. It is this equivalence that makes the cluster expansion susceptible to the standard field theory renormalization methods.

In the region defined by $q l \ll 1$ and $s-s_{c} \ll 1$, the cluster expansion to first order in $u$ can be written as follows: For convenience, each term is labeled with the letter corresponding to its graphical counterpart in Fig. 1:

$$
\begin{aligned}
\tilde{W}\left(q, t_{0}, u, l\right)= & 2 \tau_{c}^{3}\left(\frac{l}{2 \pi}\right)^{d} \int^{l^{-1}} d^{d} k \frac{1}{\left(k^{2}+t_{0}\right)^{2}} \\
& \times\left\{\frac{1^{(a)}}{(k+q)^{2}+t_{0}}+\frac{2 u}{\left(k^{2}+t_{0}\right)\left[(k+q)^{2}+t_{0}\right]} \int^{l^{-1}} d^{d} p \frac{1^{(b),(c)}}{(k+p)^{2}+t_{0}}+\frac{u}{\left[(k+q)^{2}+t_{0}\right]^{2}}\right. \\
& \times \int^{l^{-1}} d^{d} p \frac{1^{(d)}}{(k+p+q)^{2}+t_{0}}+\frac{2 u}{(k+q)^{2}+t_{0}} \int^{t^{-1}} d^{d} p \frac{1^{(o),(s)}}{\left[(k+p+q)^{2}+t_{0}\right]\left[(k+p)^{2}+t_{0}\right]} \\
& \left.+u \int^{l^{-1}} d^{d} p \frac{1^{(s)}}{\left[(k+p+q)^{2}+t_{0}\right]\left[(k+p)^{2}+t_{0}\right]^{2}}\right\}+\mathcal{O}\left(u^{2}\right) .
\end{aligned}
$$

This expansion is similar to the corresponding first order result for the linear chain. ${ }^{7}$ The distinguishing feature is the extra loop integration originating from the circularity constraint $r_{0}=r_{N}$ in the ring polymer.

\section{RENORMALIZATION AND SCALING LAWS}

The infinities which exist in perturbative field theories are now a crucial feature of the polymer cluster expansion. If the microscopic scale $l$ goes to zero, then the large momentum region of the integration causes certain of the Feynman integrals to diverge for $d \geqslant 2$ and $d \geqslant 4$. This ultraviolet catastrophe is a useful feature of the cluster expansion because it is precisely from an analysis of these divergences that the renormalization group theory is able to derive the nonperturbative scaling behavior of the theory. It will be shown that the existence of a well-defined renormalized polymer theory independent of $l$ in the limit $l=0$ is the fundamental feature of the polymer system from which emerge the scaling laws and universality. 
The structure of the polymer graphs allow a systematic study of the ultraviolet divergences. The graphs contributing to the first order cluster expansion are "super ficially" convergent for $d<5$. Superficial convergence describes their global behavior when all the integration variables go to infinity simultaneously. However, there exist integrations over a subset of the momenta, namely, the $D$ variable integrations in Eq, (2.10) labeled (b), (c), (d) and (e), (f), which diverge for $d \geqslant 2$ and $d \geqslant 4$, respectively. The source of these infinities are the divergent subdiagrams in Fig. 2. A detailed analysis of the polymer graph subdiagrams in all orders is necessary to formulate a sufficient scheme which eliminates the infinities. It can be shown that for $d>4$, the overall divergent behavior of the theory becomes worse as the or der of the diagrams increases. This situation renders the theory for $l=0$ meaningless because it is not possible to eliminate the divergences to all orders. When $d=4$, the divergent behavior is the same in all orders and the theory is said to be renormalizable. In four dimensions and less, there exists a well-defined procedure which renders the cluster expansion finite to all orders.

Renormalization is the systematic procedure whereby the infinite parts of the diagrams are subtracted away in each order and absorbed into a redefinition of certain variables in the theory. It can be shown ${ }^{24}$ from the graphical structure of the polymer field theory that a redefinition of four quantities in the theory is necessary to eliminate the divergences to all orders for $d \leqslant 4$. The four parameters, denoted by $t_{0}\left(s_{c}\right), z_{1}, z_{2}$, and $z_{3}$, are able to absorb the infinite parts if the following redefinitions are made:

$$
\begin{array}{ll}
t(s)=t_{0}(s)-t_{0}\left(s_{c}\right) & u_{R}=s(d) z_{3} u, \\
t_{R}=z_{2} t, & \bar{W}_{R}=z_{1} \bar{W} .
\end{array}
$$

The parameters $z_{1}, z_{2}$, and $z_{3}$ are dimensionless and known as the renormalization constants. The factor $s(d)$ is the surface area of the unit sphere in $d$ dimensions and is included in the definition of $u_{R}$ for later convenience. The statement of the renormalizability of the theory is the following: Having re-expressed the theory in terms of the shifted chemical potential $t=\tau_{c}(s$ $\left.-s_{c}\right)$, there exists $z_{1}, z_{2}$, and $z_{3}$ independent of $q$ and $t$ such that the expansion for $z_{1} W(q, t, u, l)$, when expressed as a function of $t_{R}=z_{2} t$ and $u_{R}=\$(d) z_{3} u$, is finite in all orders for $l=0$ and $d \leqslant 4$.

We first consider the change of variable from $t_{0}(s)$ to $t(s)$. This is responsible for eliminating a divergence associated with the intramolecular "self-energy" subdiagram in Fig. 2(a). This is also a very natural change of variable because $t(s)$ provides a convenient measure of the distance away from the critical point. It should be noted that the critical chemical potential $s_{c}$, which is determined from the condition $\left[\tilde{Q}\left(q=0, s_{c}, u, l\right)\right]^{-1}=0$, depends on $u$ and $l$. When expressed in terms of $t$, the cluster expansion from $\mathrm{Eq}$. (2, 10) becomes

$$
\begin{aligned}
& \tilde{W}(q, t, u, l)=\omega \int^{t^{-1}} d^{d} k \frac{1}{\left(k^{2}+t\right)^{2}}\left\{\frac{1}{(k+q)^{2}+t}+\frac{2 u}{\left(k^{2}+t\right)\left[(k+q)^{2}+t\right]} \int^{l^{-1}} d^{d} p\left[\frac{1}{(k+p)^{2}+t}-\frac{1}{p^{2}}\right]\right. \\
& +\frac{u}{\left[(k+q)^{2}+t\right]^{2}} \int^{l^{-1}} d^{d} p\left[\frac{1}{(k+p+q)^{2}+t}-\frac{1}{p^{2}}\right]+\frac{2 u}{(k+q)^{2}+t} \int^{l^{-1}} d^{d} p \frac{1}{\left[(k+p+q)^{2}+t\right]\left[(k+p)^{2}+t\right]} \\
& \left.+u \int^{l-1} d^{d} p \frac{1}{\left[(k+p+q)^{2}+t\right]\left[(k+p)^{2}+t\right]^{2}}\right\}+o\left(u^{2}\right)
\end{aligned}
$$

where $\omega \equiv 2 \tau_{c}^{3}(l / 2 \pi)^{d}$. The subtractions occuring in the integrals indicate that this change of variables is responsible for removing the quadratic divergence at $d=4$ associated with the subdiagram in Fig. 2 (a). In the domain $d \leqslant 4$, the only ultraviolet infinities that remain in this expression are logarithmic divergences at $d=4$. If $d<4$, then it is safe for the boundaries of the integrations to recede to infinity. Then the cluster expansion can be written in the simplified form

$$
\tilde{W}(q, t, u)=-\omega\left[\left(\frac{1}{2}+u I_{1}(q, t)+\frac{1}{2} u I_{1}(0, t)\right) \frac{\partial I_{1}(q, t)}{\partial t}+\frac{1}{2} u t I_{2}(t) \frac{\partial^{2} I_{1}(q, t)}{\partial t^{2}}+o\left(u^{2}\right)\right],
$$

where

$$
\begin{aligned}
& I_{1}(q, t)=\int d^{d} p \frac{1}{\left[(p+q)^{2}+t\right]\left(p^{2}+t\right)}, \\
& I_{2}(t)=\int d^{d} p \frac{1}{p^{2}\left(p^{2}+t\right)} .
\end{aligned}
$$

The absence of ultraviolet divergences in the cluster expansion for $d<4$ is still not sufficient to allow the expansion to be used as a perturbative description of the self-repelling polymer in three dimensions. This is due to the existence of infrared divergences in the theory.
From dimensional analysis, the general structure of the cluster expansion can be written in the following form:

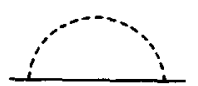

(a)

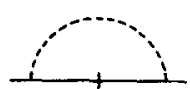

(b)
FIG. 2. The divergent subdiagrams contributing in first order to the polymer field theory. 


$$
\tilde{W}(q, t, u, l)=\sum_{n=0}^{\infty} J_{n}\left(q l, t l^{2}, d\right)\left(u t^{(d / 2)-2}\right)^{n}
$$

$J_{n}$ is a dimensionless function depending on the Feynman graphs of order $n$ and is free of ultraviolet divergences for $d<4$. The effective coupling in the expansion is the dimensionless parameter $u t^{(d / 2)-2} \sim \tilde{f}\left(t l^{2}\right)^{(d / 2)-2}$. For $d<4$ and fixed repulsion strength $\tilde{f}$, this cluster expansion is meaningless in the critical region $(q l \ll 1$, $\left.t l^{2} \ll 1\right)$ because every order in the expansion is arbitrarily larger than a lower order. Thus, the problem of the self-repelling polymer must be solved using concepts and methods that go beyond the perturbation theory. These methods are provided by the renormalization group theory. The renormalizability of the perturbation theory at $d=4$, via the existence of $z_{1}, z_{2}$, and $z_{3}$, is the feature of the polymer theory that allows the renormalization group analysis to derive the nonperturbative scaling behavior for the physical dimension $d=3$.

The renormalization group equation is a consequence of the renormalizability of the theory that leads to the general structure of the nonperturbative properties of the theory. This equation is a partial differential equation for the function $\bar{W}_{R}$ whose solution represents the infinite sum of the renormalized cluster expansion. It is convenient to define the dimensionless coupling constants $g$ and $g_{R}$ :

$$
\begin{aligned}
& g \equiv \kappa^{d-4} u, \\
& g_{R} \equiv \kappa^{d-4} u_{R},
\end{aligned}
$$

where $\kappa$ is an arbitrary scale parameter with the dimensions of a momentum. The renormalization group equation for the function $\tilde{W}_{R}\left(q, t_{R}, g_{R}, \kappa\right)$ is based on a statement of the independence of the bare (physical) function $\bar{W}(q, t, u, l)$ with respect to the unphysical parameter $k$ :

$$
\left(\kappa \frac{\partial}{\partial \kappa}\right)_{t, u, l} \tilde{W}(q, t, u, l)=0 .
$$

The renormalizability of the function $\tilde{W}(q, t, u, l)$ enables this equation to be expressed in terms of the renormalized quantities in the limit $l=0$;

$$
\begin{aligned}
{\left[\kappa \frac{\partial}{\partial \kappa}+\beta\left(g_{R}\right) \frac{\partial}{\partial g_{R}}+\gamma_{1}\left(g_{R}\right)\right.} & \left.+\gamma_{2}\left(g_{R}\right) t_{R} \frac{\partial}{\partial t_{R}}\right] \\
& \times \bar{W}_{R}\left(q, t_{R}, g_{R}, \kappa\right)=0,
\end{aligned}
$$

where

$$
\begin{aligned}
& \beta\left(g_{R}\right)=\left(\frac{\partial g_{R}}{\partial \ln \kappa}\right)_{u}, \\
& \gamma_{1}\left(g_{R}\right)=-\left(\frac{\partial \ln z_{1}}{\partial \ln \kappa}\right)_{u}, \\
& \gamma_{2}\left(g_{R}\right)=\left(\frac{\partial \ln z_{2}}{\partial \ln \kappa}\right)_{u} .
\end{aligned}
$$

As an expansion in $g_{R}$, the functions $\beta\left(g_{R}\right), \gamma_{1}\left(g_{R}\right)$, and $\gamma_{2}\left(g_{R}\right)$ are well defined for $d \leqslant 4$ despite the divergences at $d=4$ contained in $z_{1}, z_{2}$, and $z_{3}$. This significant feature is a consequence of the renormalizability of the theory for $d \leqslant 4 .{ }^{24}$

The renormalization group equation provides the means for transcending the perturbation theory to derive properties which cannot be extracted from the finite order cluster expansion. In particular, it can be used to prove the existence of scaling behavior in the theory. The universal behavior is expected to occur in the asymptotic regime corresponding to a large ring $\left(t l^{2} \ll 1\right)$ and large distances $(q l \ll 1)$. From the definition of $\beta\left(g_{R}\right)$ in Eq. (3.9), it can be shown that this asymptotic behavior of the bare theory is governed by the zeros of $\beta\left(g_{R}\right)$. For the special value of the renormalized coupling $g_{R}^{*}$ such that $\beta\left(g_{R}^{*}\right)=0$, the renormalization group equation simplifies and has a solution that can be written in the following form:

$$
\bar{W}_{R}\left(q, t_{R}, g_{R}^{*}, \kappa\right)=\omega \kappa^{d-6}\left(\frac{t_{R}}{\kappa^{2}}\right)^{\nu d-3} F\left(\frac{q}{\kappa}\left(\frac{t_{R}}{\kappa^{2}}\right)^{-\nu}\right),
$$

where $\nu$ is given by the relation

$$
\gamma_{2}\left(g_{R}^{*}\right)=2-\frac{1}{v}
$$

$F(x)$ is an undetermined scaling function and $\nu$ is the exponent which appears in the scaling law for the radius of gyration $\left\langle R_{G}^{2}\right\rangle \sim N^{2 \nu}$.

The scaling behavior of the structure function $S_{N}(q)$ is readily obtained from Eq. (3.10) by a transformation back to the bare theory in the canonical ensemble. The result is

$$
S_{N}(q)=a_{1} N^{2} \frac{e^{s_{e} N} N^{-\nu d}}{Z_{N}} G\left(a_{2} q N^{\nu}\right) .
$$

The constants $a_{1}$ and $a_{2}$ depend only on the microscopic parameters $\vec{f}$ and $l$ in the theory. The scaling function $G(y)$ remains undetermined. The partition function $Z_{N}$ for a large self-avoiding ring polymer is ${ }^{25}$

$$
Z_{N}=a_{3} e^{s_{c N}^{N}} N^{-\nu d},
$$

where $a_{3}$ is independent of $N$. The exponent $\gamma$, which characterizes the partition function for a linear chain $\left(Z_{N} \sim e^{s_{c} N} N^{y-1}\right),{ }^{25}$ does not appear in the ring function. Instead, there is the suppression factor $N^{-\nu d}$ which reflects the circularity constraint in the ring polymer. This $Z_{N}$ allows the structure function for the ring poly mer in Eq. (3.12) to be written as

$$
S_{N}(q)=N^{2} f_{\mathbf{s}}\left(B q N^{\nu}\right) \text {. }
$$

$f_{s}(x)$ is a universal scaling function such that $f_{s}(0)=1$ and $B$ depends on the microscopic structure of the theory. This scaling law has the same form as the corresponding scaling law for the linear chain ${ }^{7}$ although the scaling functions are different.

The scaling relations and their universality are a concrete realization of the renormalization symmetry of a ring polymer. This symmetry is a consequence of the renormalizability of the polymer theory which establishes the existence of an underlying renormalized theory that is independent of the microscopic length scale $l$. The renormalization group equation is an expression of this symmetry from which emerge only the general structure of the scaling laws. The renormalization group equation does not provide the information on more detailed properties of the polymer that is con- 
tained in the scaling function and the exponent. To calculate these quantities, it is necessary to know the explicit values of the renormalization constants $z_{i}$ as determined from the renormalization of the cluster expansion at $d=4$. This renormalization requires the use of more detailed field theoretical methods that are described in the next section.

\section{EXPANSION IN 4-d AND THE SCALING FUNCTION}

The cluster expansion contains the information necessary to calculate the scaling function and the exponent. To extract this information from the ill-behaved perturbation series, we renormalize the cluster expansion using the methods of dimensional regularization and minimal subtraction renormalization. This renormalization program involves generalizing the Feynman diagrams to noninteger dimensions, expanding in $\epsilon \equiv 4-d$ and then removing the ultraviolet divergences which appear at $\epsilon=0$. This procedure leads to a renormalized coupling $g_{R}^{*}$ of order $\epsilon$ which turns the meaningless perturbation series in $u$ into a meaningful perturbation series in $\epsilon$. The expansion in $\epsilon$ is a device with which the calculations can be systematically controlled. In particular, it allows us to calculate the first order $\epsilon$ expansions of the scaling function and the exponent using only the zeroth and first order polymer graphs.

Dimensional regularization ${ }^{17,24}$ is a procedure whereby the theory is generalized to allow for noninteger values for the dimension $d$. This is accomplished by an analytic continuation of the Feynman integrals into the complex $d$ plane. This mathematical maneuver is useful because the resulting integrals are now smoothly varying functions of the variable $d$ and thus susceptible to an expansion in $\epsilon$. The singularities which exist in the integrals for $l=0$ and $d=4$ manifest themselves as poles in €. As an example, consider the integrals in Eq. (3.4) that are required to calculate the cluster expansion to first order. The $d$-dimensional integrations may be computed using a standard prescription. ${ }^{24}$ The result is

$$
\begin{aligned}
& I_{1}(q, t)= \frac{1}{2} s(d) \Gamma\left(\frac{d}{2}\right) \Gamma\left(2-\frac{d}{2}\right) \int_{0}^{1} \\
& \times d x\left[t+x(1-x) q^{2}\right]^{d / 2-2}, \\
& I_{2}(t)=\frac{1}{2} s(d) \Gamma\left(\frac{d}{2}\right) \Gamma\left(2-\frac{d}{2}\right)\left(\frac{d}{2}-1\right)^{-1} t^{d / 2-2} .
\end{aligned}
$$

The factor $s(d)$ arises from the angular integrations. The Euler gamma function $\Gamma(2-d / 2)$ reflects the singularity when $d=4$. The desired $\epsilon$ expansion is obtained by inserting $d=4-\epsilon$ into these expressions and expanding the $\epsilon$-dependent quantities. The result is

$$
\begin{aligned}
& I_{1}(q, t)= \kappa^{-\epsilon} s(d) \\
& \times\left\{\frac{1}{\epsilon}-\frac{1}{2}-\frac{1}{2} \int_{0}^{1} d x \ln \left[\bar{t}+x(1-x) \bar{q}^{2}\right]+\mathcal{O}(\epsilon)\right\} \\
& \equiv \kappa^{-\epsilon} \delta(d) \overline{I_{1}}(\bar{q}, \bar{t}) \\
& I_{2}(t)=\kappa^{-\epsilon} s(d)\left[\frac{1}{\epsilon}-\frac{1}{2} \ln \bar{t}+\mathcal{O}(\epsilon)\right] \equiv \kappa^{-\epsilon} s(d) \bar{I}_{2}(\bar{t})
\end{aligned}
$$

where $\bar{q} \equiv q / \kappa$ and $\bar{t} \equiv t / \kappa^{2}$ and for convenience we have defined the dimensionless integrals $\bar{I}_{1}$ and $\bar{I}_{2}$. The $\epsilon$ expansion has succeeded in isolating the singular part of the integral. This part is denoted by $\left(\overline{V_{1}}\right)_{\mathrm{sing}}=\left(\overline{I_{2}}\right)_{\mathrm{sing}}$ $=1 / \epsilon$. This is an extremely useful feature of the dimensionally regularized theory which allows an easy removal of the infinities.

Minimal subtraction ${ }^{17,24}$ is a natural and efficient renormalization scheme designed for the dimensionally regularized theory. In this scheme, the renormalization constants $z_{1}, z_{2}$, and $z_{3}$ are determined by the requirement that the dimensional poles be canceled in every order in the perturbation expansion. We demonstrate this procedure to first order using the cluster expansion of $\bar{W}(q, t, u)$ given in Eq. (3.3). The renormalization constants $z_{i}$ are dimensionless functions of $g_{R}$ and $\epsilon$ which can be written as

$$
\begin{aligned}
& z_{1}\left(g_{R}, \epsilon\right)=1+\sum_{n=1}^{\infty} a_{n}(\epsilon) g_{R}^{n}, \\
& z_{2}\left(g_{R}, \epsilon\right)=1+\sum_{n=1}^{\infty} b_{n}(\epsilon) g_{R}^{n}, \\
& z_{3}\left(g_{R}, \epsilon\right)=1+\sum_{n=1}^{\infty} c_{n}(\epsilon) g_{R}^{n} .
\end{aligned}
$$

The coefficients $a_{i}(\epsilon), b_{i}(\epsilon)$, and $c_{i}(\epsilon)$ are the absorbers of the dimensional singularities. Using these expansions for the renormalization constants, the function $z_{1} \tilde{W}(q, t$, $u$ ) can be expressed in terms of $t_{R}=z_{2} t$ and $g_{R}=s(d) z_{3} g$. The result is

$$
\begin{aligned}
& \bar{W}_{R}\left(q, t_{R}, g_{R}, \kappa\right)=-\omega \kappa^{d-6} s(d)\left[\frac{1}{2} \frac{\partial \bar{I}_{1}\left(\bar{q}, \bar{t}_{R}\right)}{\partial \bar{t}_{R}}+g_{R}\left(\frac{1}{2} a_{1}(\epsilon)\right.\right. \\
& \left.\quad+\bar{I}_{1}\left(\bar{q}, \bar{t}_{R}\right)+\frac{1}{2} \bar{I}_{1}\left(0, \bar{t}_{R}\right)\right) \frac{\partial \bar{I}_{1}\left(\bar{q}, \bar{t}_{R}\right)}{\partial \bar{t}_{R}} \\
& \left.\quad+\frac{1}{2} g_{R} \bar{t}_{R}\left(\bar{V}_{2}\left(\bar{t}_{R}\right)-b_{1}(\epsilon)\right) \frac{\partial^{2} \bar{I}_{1}\left(\bar{q}, \bar{t}_{R}\right)}{\partial \bar{t}_{R}^{2}}+O\left(g_{R}^{2}\right)\right] .
\end{aligned}
$$

In this expression, $\bar{I}_{1}\left(\bar{q}, \bar{t}_{R}\right)$ and $\bar{I}_{2}\left(\bar{t}_{R}\right)$ are the dimensionally regularized integrals appearing in Eq. (4.2) and $\bar{t}_{R} \equiv t_{R} / \kappa^{2}$. The requirement that the dimensional poles be minimally subtracted is easily realized by choosing

$$
\begin{aligned}
& a_{1}(\epsilon)=-3\left(\overline{I_{1}}\right)_{\mathrm{sing}}=-\frac{3}{\epsilon}, \\
& b_{1}(\epsilon)=\left(\overline{I_{2}}\right)_{\sin \theta}=\frac{1}{\epsilon} .
\end{aligned}
$$

Using these values for $z_{1}$ and $z_{2}$, the renormalized cluster expansion to first order in $g_{R}$ is finite for $d \leqslant 4$.

The renormalized function $\tilde{W}_{R}\left(q, t_{R}, g_{R}, \kappa\right)$ must now be evaluated at the "fixed point" coupling $g_{R}^{*}$ for comparison with the scaling law in Eq. (3.10). Recall that $g_{R}^{*}$ is the zero of the function $\beta\left(g_{R}\right)$ defined in Eq. (3.9), which can be written as

$$
\beta\left(g_{R}\right)=-\epsilon\left(\frac{\partial \ln g}{\partial g_{R}}\right)^{-1} .
$$

Renormalization of the cluster expansion to first order in $g_{R}$ does not provide the necessary information, namely, $z_{3}\left(g_{R}, \epsilon\right)$, to calculate $\beta\left(g_{R}\right)$. This is because the divergent subdiagrams which determine $z_{3}$ to first order do 
not exist in the first order cluster expansion. These diagrams are represented in the polymer vertex function $\Gamma^{(4)}\left(p_{1}, p_{2}, p_{3}, p_{4}\right)$. This function is defined as the set of the irreducible polymer graphs to which four external lines carrying momenta $p_{1}, p_{2}, p_{3}$, and $p_{4}$ may be attached. An irreducible graph is one which cannot be separated into two disjoint pieces by cutting a single polymer line. The diagrams contributing to $\Gamma^{(4)}$ to second order in $u$ are displayed in Fig. 3. The corresponding algebraic expansion is

$$
\Gamma^{(4)}\left(p_{i}, t, u\right)=\tau_{c}^{-2}\left(\frac{l}{2 \pi}\right)^{-d} u\left\{1+u\left[I_{1}\left(p_{1}+p_{3}, t\right)+I_{1}\left(p_{1}+p_{4}, t\right)+I_{1}\left(p_{3}+p_{4}, t\right)+I_{1}\left(p_{1}+p_{2}, t\right)\right]+o\left(u^{2}\right)\right\} .
$$

The renormalization of this expansion by subtracting the dimensional poles at $d=4$ is sufficient to determine the renormalization constant $z_{3}$. The result is

$$
z_{3}\left(g_{R}, \epsilon\right)=1+\frac{4}{\epsilon} g_{R}+o\left(g_{R}^{2}\right)
$$

This first order result for $z_{3}$ enables one to calculate the fixed point coupling $g_{R}^{*}$ using Eq. (4.6). The exponent $\nu$ can be calculated using its definition [Eqs. (3.11) and (3.9)] and the value of $z_{2}[E q$. (4.5)]. To first order in $\epsilon$, the result is

$$
\begin{aligned}
& g_{R}^{*}=-\frac{\epsilon}{4}+\mathcal{O}\left(\epsilon^{2}\right), \\
& \nu=\frac{1}{2}+\frac{\epsilon}{16}+\mathcal{O}\left(\epsilon^{2}\right) .
\end{aligned}
$$

Substituting this value for $g_{R}^{*}$ into Eq. (4.4) yields the first order $\epsilon$ expansion of $\tilde{W}_{R}\left(q, t_{R}, g_{R}^{*}, k\right)$ :

$$
\begin{aligned}
\tilde{W}_{R}\left(q, t_{R}, g_{R}^{*}, \kappa\right)= & \frac{1}{4} \omega \kappa^{d-6} s(d) \int_{0}^{1} d x\left[\bar{t}_{R}+x(1-x) \bar{q}^{2}\right]^{-1} \\
& \times\left(1+\epsilon\left\{\frac{1}{8}\left(\ln \bar{t}_{R}-1\right)+\frac{1}{4} \int_{0}^{1} d y \ln \left[\bar{t}_{R}+y(1-y) \bar{q}^{2}\right]-\frac{1}{2} \ln \left[\bar{t}_{R}+x(1-x) \bar{q}^{2}\right]\right.\right. \\
& \left.\left.-\frac{1}{8} \bar{t}_{R} \ln \bar{t}_{R}\left[\bar{t}_{R}+x(1-x) \bar{q}^{2}\right]^{-1}\right\}+\mathcal{O}\left(\epsilon^{2}\right)\right) .
\end{aligned}
$$

This renormalized cluster expansion is the perturbative (in $\epsilon$ ) representation of the scaling law for the function $W_{R}\left(q, t_{R}, g_{R}^{*}, \kappa\right)$ in Eq. (3.10). A comparison of the cluster expansion and the scaling law, both expanded in $q$ and $\epsilon$, is sufficient to determine the scaling function $F(x)$ of $\tilde{W}_{R}\left(q, t_{R}, g_{R}^{*}, \kappa\right)$. To first order in $\epsilon$, one finds

$$
F(x)=\left.\sum_{n=0}^{\infty} \frac{1}{(2 n) !} \frac{d^{2 n} F(x)}{d x^{2 n}}\right|_{x=0} x^{2 n}
$$

where

$$
\left.\frac{1}{(2 n) !} \frac{d^{2 n} F(x)}{d x^{2 n}}\right|_{x=0}=s(d) \frac{(-1)^{n}}{4} B(n)\left(1-\frac{\epsilon}{8}\left\{1+2 \sum_{m=0}^{n-1} \frac{1}{n-m}\left[\frac{B(m) B(n-m)}{B(n)}-2\right]\right\}+o\left(\epsilon^{2}\right)\right)
$$

and

$$
B(a) \equiv \frac{(a !)^{2}}{(2 a+1) !}
$$

\section{RESULTS AND DISCUSSION}

The structure function $S_{N}(q)$ of a ring polymer with $N$ monomers can be obtained from a transformation of the grand canonical correlations, as defined by the function $\tilde{W}(q, t, u, l)$, into the canonical ensemble. Using the scaling law in Eq. (3.10), this bare function can be written for $q l \ll 1, t l^{2} \ll 1$ as

$$
\begin{aligned}
W(q, t, u, l) & =c_{1} t^{d \nu-3} F\left(c_{2} q t^{-\nu}\right) \\
& =\sum_{n=0}^{\infty} a_{2 n} t^{d \nu-3-2 \nu n} q^{2 n},
\end{aligned}
$$

where

$$
\begin{aligned}
& \tilde{W}(q, t, u, l)=\sum_{N=0}^{\infty} \exp (-s N) \tilde{W}(q, N, u, l) \\
& \left.a_{2 n} \equiv c_{1} c_{2}^{2 n} \frac{1}{(2 n) !} \frac{d^{2 n} F(x)}{d x^{2 n}}\right|_{x=0} .
\end{aligned}
$$

The constants $c_{1}$ and $c_{2}$ depend on $\tilde{f}$ and $l$ and are thus nonuniversal. The representation of $\tilde{W}(q, t, u, l)$ in the canonical ensemble is $W(q, N, u, l)$ and the connection between these functions is given by
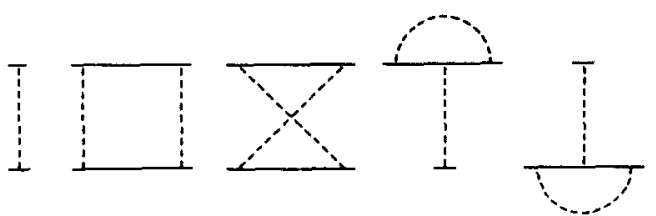

FIG. 3. The diagrams for the vertex function $\Gamma^{(4)}$ to second order. 


$$
\equiv \sum_{N=0}^{\infty} \exp (-s N) \sum_{n=0}^{\infty} \boldsymbol{x}_{2 n}(N) q^{2 n},
$$

where $t=\tau_{c}\left(s-s_{c}\right)$. Thus, the desired connection between the canonical $\alpha_{2 n}$ and the grand canonical $a_{2 n}$ coefficients can be obtained from an inversion of the Laplace transformation. For large $N$, the result is

$$
x_{2 n}(N)=a_{2 n} \tau_{c}^{d \nu-3-2 \nu n} \exp \left(s_{c} N\right) \frac{N^{2-d u+2 n \nu}}{\Gamma(3-d \nu+2 n \nu)} .
$$

The structure function

$$
S_{N}(q)=Z_{N}^{-1} \tilde{W}(q, N, u, l)
$$

can now be represented by an expansion that is consistent with the scaling law derived in Eq. (3.14). Using Eqs. (3.13) and (5.4), the structure function of the ring polymer can be written as

$$
S_{N}(q)=N^{2} \sum_{n=0}^{\infty} \Omega_{2 n} Q^{2 n}
$$

where the scaled variable $Q=B q N^{\nu}$ is given by

$$
Q^{2}=\frac{a_{2}}{a_{0}} \tau_{c}^{-2 \nu} \frac{\Gamma(3-d \nu)}{\Gamma(3-d \nu+2 \nu)} q^{2} N^{2 \nu}
$$

and coefficients $\Omega_{2 n}$ in the series are

$$
\Omega_{2 n}=\frac{a_{2 n}}{a_{0}}\left(\frac{a_{0}}{a_{2}}\right)^{n} \frac{[\Gamma(3-d \nu+2 \nu)]^{n}}{\Gamma(3-d \nu+2 n \nu)[\Gamma(3-d \nu)]^{n-1}} .
$$

Having already calculated the grand canonical coefficients $a_{2 n}$ in Eq. (4.11), one may readily evaluate the quantities $Q$ and $\Omega_{2 n}$. The dependence on the microscopic parameters resides in $Q$, thus rendering it a nonuniversal quantity. A cancellation of the microscopic parameters in $\Omega_{2 n}$ leaves it a universal function of $\epsilon$ :

$$
\begin{aligned}
\Omega_{2 n}= & \frac{[6 \Gamma(3-d \nu+2 \nu)]^{n} B(n)}{\Gamma(3-d \nu+2 n \nu)[\Gamma(3-d \nu)]^{n-1}} \\
& \times\left(1-\frac{\epsilon}{4}\left\{n+\sum_{m=0}^{n-1} \frac{1}{n-m}\left[\frac{B(m) B(n-m)}{B(n)}-2\right]\right\}+\mathcal{O}\left(\epsilon^{2}\right)\right)
\end{aligned}
$$

For $d=4\left(\epsilon=0, \nu=\frac{1}{2}\right)$, we recover the coefficients in the expansion of the structure function for the ideal ring [Eq. (1.2)].

The representation of the structure function in terms of the scaled variable $Q$ such that $\Omega_{0}=\Omega_{2}=1$ is useful because it achieves a convenient isolation and inter pretation of the universal and nonuniversal components

TABLE I. The universal coefficients [Eq. (5.9)] in the expansion of the structure function of a ring polymer computed using the first order and the resummed $(d=3) \epsilon$ expansion of $\nu$.

\begin{tabular}{llll}
\hline$\nu$ & $\Omega_{4}$ & $\Omega_{6}$ & $\Omega_{8}$ \\
\hline$\frac{1}{2}+\frac{\epsilon}{16}$ & $\frac{3}{5}\left(1-\frac{\epsilon}{12}\right)$ & $\frac{9}{35}\left(1-\frac{11 \epsilon}{48}\right)$ & $\frac{3}{35}\left(1-\frac{101 \epsilon}{240}\right)$ \\
0.588 & $0.564\left(1-\frac{\epsilon}{12}\right)$ & $0.216\left(1-\frac{5 \epsilon}{24}\right)$ & $0.062\left(1-\frac{43 \epsilon}{120}\right)$ \\
\hline
\end{tabular}

of $S_{N}(q)$. The physical significance of $\Omega_{2 n}$ and $Q$ is independent of any particular model for the polymer and follows simply from the definition of the structure function in Eq. (1.1). Upon expanding the exponential in that expression and appropriately scaling the expansion variable so that $S_{N}(q)$ has the form $N^{2}\left(1+Q^{2}\right.$ $\left.+\Omega_{4} Q^{4}+\ldots\right)$, one finds that for $d$ dimensions

$$
S_{N}(q)=N^{2} \sum_{n=0}^{\infty} \frac{\left(\frac{d}{2}\right)^{n} \Gamma\left(\frac{d}{2}\right)}{n ! \Gamma\left(\frac{d}{2}+n\right)} \frac{\left\langle r^{2 n}\right\rangle}{\left\langle r^{2}\right\rangle^{n}}\left(-\frac{1}{d}\left\langle R_{G}^{2}\right\rangle q^{2}\right)^{n}
$$

where

$$
\left\langle r^{2 n}\right\rangle \equiv N^{-2} \sum_{i=1}^{N} \sum_{j=1}^{N}\left\langle\left|r_{i}-r_{j}\right|^{2 n}\right\rangle .
$$

Thus, the coefficients $\Omega_{2 n}$ give universal ratios of moments of the monomer distribution function. The nonuniversal part of $S_{n}(q)$ is absorbed into the scaled variable $Q$ which provides information on the radius of gyration.

The universal ratios of moments $\Omega_{2 n}$ may be computed using Eq. (5.9). The first few of these coefficients, evaluated using two different representations for the exponent $\nu$, are displayed in Table I. The existence of these alternative numerical estimates for $\Omega_{2 n}$ arises from the sensitivity of the $\epsilon$ expansion to the statistical ensemble in which it is performed. Our formalism allows one to $\epsilon$ expand either $\tilde{W}(q, N, u, l)$ or $\bar{W}(q, t, u, l)$. The resulting canonical and grand canonical $\epsilon$ expansions are related by the factor involving the gamma functions in Eq. (5.8). This conversion factor depends on the exponent $\nu$, which may be consistently expanded in $\epsilon$ to give a result that is equivalent to a direct $\epsilon$ expansion of $S_{N}(q)$. However, for obtaining estimates of $\Omega_{2 n}$ in three dimensions, it is also reasonable to choose $\nu$ as the pure number given by the resummation of its $\epsilon$ expansion for $d=3(\nu=0.588) .{ }^{26}$ Since the calculations are performed at a finite order in $\epsilon$, it is natural to use the convergence behavior of these alternative expansions as an indication of their accuracy, thereby providing a criterion for the choice of $\nu$.

A similar expansion exists for the structure function of a self-repelling linear polymer. We use the results of Witten and Schafer ${ }^{7}$ to calculate the Taylor coefficients of $S_{N}^{11 n}(q)$. To first order in $\epsilon$, the result is

$$
S_{N}^{11 \mathrm{ne}}(q)=N^{2} \sum_{n=0}^{\infty} \zeta_{2 n} X^{2 n},
$$

where

$$
X^{2}=-c_{2}^{2} \tau_{c}^{-2 \nu}\left(1+\frac{\epsilon}{96}\right) \frac{\Gamma(\gamma+2)}{\Gamma(\gamma+2+2 \nu)} q^{2} N^{2 \nu}
$$

and

$$
\begin{gathered}
\zeta_{2 n}=\frac{[\Gamma(\gamma+2+2 \nu)]^{n}}{\Gamma(\gamma+2+2 n \nu)[\Gamma(\gamma+2)]^{n-1}}\left\{1-\frac{\epsilon}{16}\left[\frac{n}{6}-1+B(n)\right.\right. \\
\left.\left.+4 \sum_{m=1}^{n-1} \frac{1}{n-m} B(n-m)\right]+o\left(\epsilon^{2}\right)\right\} .
\end{gathered}
$$

Since $\zeta_{0}=\zeta_{2}=1$, the scaled variable $X$ and the coeffi- 
TABLE II. The universal coefficients [Eq. $(5,13)]$ in the expansion of the structure function of a linear polymer computed using the first order and the resummed $(d=3) \epsilon$ expansions of $\nu$ and $\gamma$.

\begin{tabular}{lllll}
\hline$\nu$ & $\gamma$ & $\zeta_{4}$ & $\zeta_{6}$ & $\zeta_{8}$ \\
\hline$\frac{1}{2}+\frac{\epsilon}{16}$ & $1+\frac{\epsilon}{8}$ & $\frac{3}{4}\left(1-\frac{7 \epsilon}{120}\right)$ & $\frac{9}{20}\left(1-\frac{151 \epsilon}{960}\right)$ & $\frac{9}{40}\left(1-\frac{577 \epsilon}{2016}\right)$ \\
0.588 & 1.162 & $0.695\left(1-\frac{\epsilon}{160}\right)$ & $0.366\left(1-\frac{\epsilon}{64}\right)$ & $0.154\left(1-\frac{13 \epsilon}{504}\right)$ \\
\hline
\end{tabular}

cients $\zeta_{2 n}$ acquire the same physical meaning as their counterparts $Q$ and $\Omega_{2 n}$ in the ring expansion. The appearance of the exponent $\gamma$ in $S_{N}^{1 \text { ne }}(q)$ is the identifying trademark of the linear polymer. The first few of the coefficients $\zeta_{2 n}$ (universal ratios of moments) are given in Table II.

Having calculated $S_{N}(q)$ as a universal function of the variable $q^{2}\left\langle R_{G}^{2}\right\rangle \equiv q^{2} R^{2}$, several observations may be made from the results. In general, the first order $\epsilon$ expansions in the grand canonical ensemble appear to be more reliable. The convergence behavior of the $\epsilon$ expansions becomes worse as one proceeds to the higher order Taylor coefficients in the structure functions for the ring and chain. This breakdown in the $\epsilon$ expansion for large $q R$ is to be expected, since in this domain the structure functions behave like $(q R)^{-2}$ for the ideal case and $(q R)^{-1 / \nu}$ for the interacting ${ }^{4}$ case. A graph of the linear and ring polymer structure functions appears in Fig. 4. For small $q R$, the structure function for the self-repelling ring is smaller than that for the ideal ring. The same behavior has also been predicted for the linear polymer. ${ }^{7}$ A comparison of the magnitudes of the first order $\epsilon$ corrections to the ideal behavior leads to the important conclusion that the self-repulsion has a greater influence in the ring polymer than it has in the chain. This is an indication of the significance of the circularity constraint in determining the function of circular DNA in biological systems. It also suggests that excluded volume effects in a ring polymer are more susceptible to experimental detection than corresponding effects in a chain. It should be noted that a more quantitative confirmation of these predictions can only be realized from a calculation involving higher order in $\epsilon$. However, it is expected that the $\epsilon$ expansion is an asymptotic series which provides reliable results in first and second order. ${ }^{27}$

The radius of gyration is a convenient measure of the size of a polymer. It is known ${ }^{18}$ that the mean square radius of gyration of an ideal linear polymer is twice that of an ideal ring polymer having the same number of monomers. We investigate the influence of the selfrepulsion on this ideal behavior. Since the radius of gyration is the correlation length (second moment) of the monomer density correlation function $\langle\rho(0) \rho(r)\rangle$, the following ratio may be constructed:

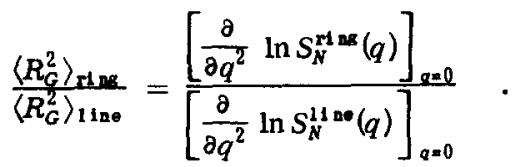

This ratio is readily calculated using the results for the structure functions of the ring and chain. One finds that

$$
\frac{\left\langle R_{G}^{2}\right\rangle_{\text {dix }}}{\left\langle R_{G}^{2}\right\rangle_{1 \mathrm{in}}}=\frac{\Gamma(3-d \nu) \Gamma(\gamma+2+2 \nu)}{\Gamma(3-d \nu+2 \nu) \Gamma(\gamma+2)} \frac{1}{6}\left[1+\frac{23}{96} \epsilon+\mathcal{O}\left(\epsilon^{2}\right)\right] .
$$

The universality of this ratio is a consequence of the cancellation of the microscopic parameters in the radii of gyrations. Using the $\epsilon$ expansions for the exponents $\nu$ and $\gamma$, the ratio is

$$
\frac{\left\langle R_{G}^{2}\right\rangle_{\text {ox }}}{\left\langle R_{G}^{2}\right\rangle_{11 \mathrm{no}}}=\frac{1}{2}\left[1+\frac{13}{96} \epsilon+O\left(\epsilon^{2}\right)\right] .
$$

Unlike the ratios of moments $\Omega_{2 n}$ and $\zeta_{2 n}$, the first order $\epsilon$ expansion of the radius of gyration ratio has a weaker $d$ dependence in the canonical ensemble. For $d=4$, we recover the ratio for ideal polymers. In three dimensions, the ratio is 0.568 . Thus, in the presence of the self-repulsion, the swelling of a ring polymer relative to its ideal state is approximately $13 \%$ greater than the swelling of a linear polymer. This

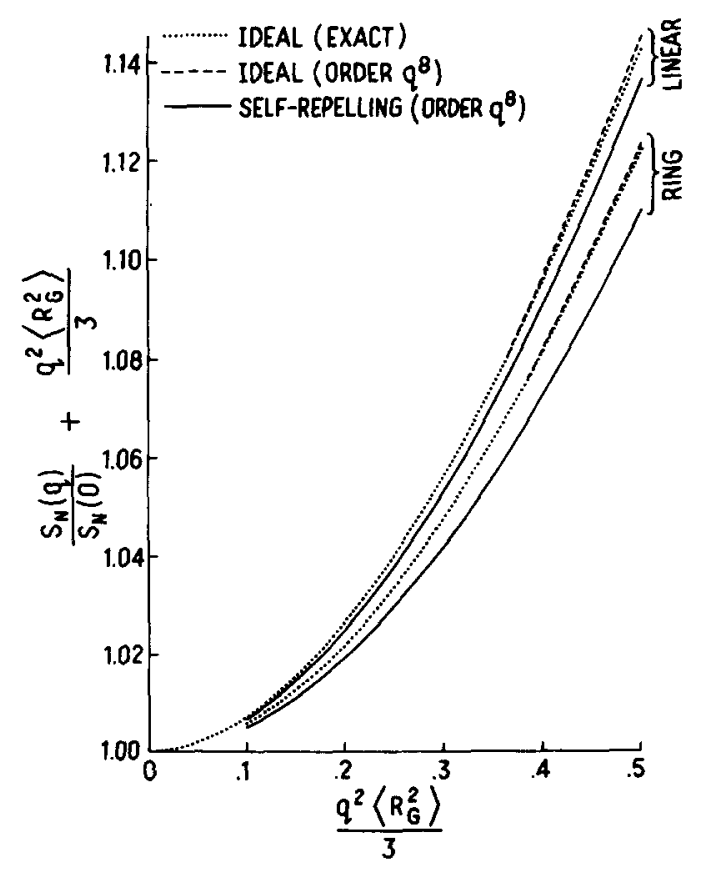

FIG. 4. The scaled structure functions for the ideal and selfrepelling linear and ring polymers. The self-repelling curves are computed using the expansion of $S_{N}(q)$ to order $q^{8}$ and setting $d=3(\nu=0.588, \gamma=1.162)$ in the coefficients. An indication of the error in truncating the expansion at this order is provided by the dashed curves. 
behavior is consistent with the prediction made above that a ring polymer exhibits properties having a greater sensitivity to the self-repulsion.

Computer simulation of the polymer statistics can also provide a prediction for the radius of gyration ratio. The off-lattice Monte Carlo study ${ }^{19}$ uses the direct method for generating self-avoiding rings having up to $N=99$ bonds. However, reliable values for the radius of gyration were obtained for rings with $N<50$ due to the difficulty in generating a sufficient number of the larger rings for statistical averaging. (Only one ring was 99 bonds was produced.) The values for the ring-chain radius of gyration ratio were extrapolated to infinite $N$ to find the limit $\mathbf{0 . 5 5 9}$. This is within $2 \%$ of the $\epsilon-\mathrm{ex}-$ pansion prediction. The recent Monte Carlo computer study ${ }^{20}$ of ring polymers using the dimerization method is a more efficient technique for generating a large number of rings with any number of bonds. In this study the radius of gyration of rings and chains containing up to 64 bonds has been calculated using very large sample sizes. In a continuation of this numerical study, ${ }^{21}$ the generation of perfect (undistorted) and larger ( $N=128$ and 256) rings and an extrapolation to infinite $N$ give a radius of gyration ratio within $0.2 \%$ of the theoretical prediction 0.568 .

The dimensions of cyclic and linear poly(dimethylsiloxane) have been measured ${ }^{22}$ using small angle neutron scattering. In this experiment, the dilute polymer solution is at a temperature of $292 \mathrm{~K}$ and contains the solvent benzene- $d_{6}$. The polymers are characterized as having a $z$-average number of bonds in the region $N_{z}$ $=500$ and as being sufficiently monodisperse to allow a direct comparison with the theoretical prediction. The experimental value for the radius of gyration ratio is $0.526 \pm 0.05$ which is consistent with the 6 -expansion result.

\section{ACKNOWLEDGMENT}

It is a pleasure to thank Professor T. A. Witten, Jr. for many helpful discussions.

${ }^{1}$ K. G. Wilson and J. B. Kogut, Phys. Rev. C 12, 75 (1974).

${ }^{2}$ P. G. de Gennes, Phys. Lett. A 38, 339 (1972).

${ }^{3} \mathrm{~J}$. des Cloizeaux, J. Phys. (Paris) 36, 281 (1975).

${ }^{4} \mathrm{~L}$. Schäfer and T. A. Witten, J. Chem. Phys. 6B, 2121 (1977).

${ }^{5}$ T. A. Witten and L. Schäfer, J. Phys. A 11, 1843 (1978).

${ }^{6} \mathrm{~J}$. des Cloizeaux, J. Phys. (Paris) 41, 223 (1980).

'T. A. Witten and L. Schäfer, J. Chem. Phys. 74, 2582 (1981).

${ }^{8} \mathrm{H}$. Yamakawa, Modem Theory of Polymer Solutions (Harper and Row, New York, 1971).

${ }^{9}$ M. J. Fixman, J. Chem. Phys. 23, 1656 (1955).

${ }^{10}$ J. des Cloizeaux, J. Phys. (Paris) 42, 635 (1981).

${ }^{11}$ Y. Oono, T. Ohta, and K. F. Freed, J. Chem. Phys. 74, 6458 (1981).

${ }^{12} J$. Vinograd and J. Lebowitz, J. Gen. Physiol. 48, 103 (1966).

${ }^{13}$ M. Hayashi, M. N. Hayashi, and S. Splegelman, Proc. Natl. Acad. Sci. USA 51, 351 (1964).

${ }^{14} \mathrm{~K}$. Dodgson, D. Sympson, and J. A. Semlyen, Polymer 10, 1285 (1978).

${ }^{15}$ D. Geiser and H. Hocker, Macromolecules 13, 653 (1980).

${ }^{16}$ E. F. Casassa, J. Polym. Sci. Part A. 3, 605 (1965).

${ }^{17} \mathrm{G}$. $t$ 'Hooft and M. Veltman, Dlagrammar CERN report 73-9, 1973.

${ }^{18}$ B. H. ZImm and W. H. Stockmayer, J. Chem. Phys. 17, 1301 (1949).

${ }^{19} \mathrm{~W}$. Bruns and J. Naghizadeh, J. Chem. Phys. 65, 747 (1976).

${ }^{20}$ Y. Chen, J. Chem. Phys. 74, 2034 (1981).

${ }^{21}$ Y. Chen, J. Chem. Phys. 75, 5160 (1981).

${ }^{22}$ J. S. Higgins, K. Dodgson, and J. A. Semlyen, Polymer 20, 553 (1979).

${ }^{23} \mathrm{~J}$. Mayer and M. G. Mayer, Statistical Mechanics (Whley, New York, 1940).

${ }^{24} \mathrm{D}$. J. Amit, Field Theory, the Renormalization Group and Critical Phenomena (MoGraw-Hill, New York, 1978).

${ }^{25} \mathrm{~J}$. des Cloizeaux. Phys. Rev. A 10, 1665 (1974).

${ }^{26}$ J. C. Le Guillou and J. Zinn-Justin, Phys. Rev. B 21, 3979 (1980).

${ }^{27} \mathrm{D}$. J. Wallace, in Phase Transitions and Critical Phenomena, edited by C. Domb and M. S. Green (Academic, New York, 1976), Vol. 6. 